\title{
Ansiedad ante los exámenes. Qué se evalúa y cómo?
}

\author{
Luis Furlan ${ }^{1 *}$ \\ * Laboratorio de Evaluación Psicológica y Educativa. Facultad de Psicología, Universidad Nacional \\ de Córdoba
}

\begin{abstract}
Resumen. En este trabajo se revisa un conjunto de instrumentos de medición de "ansiedad ante los exámenes", una reacción emocional que las personas presentan ante situaciones en las que sus aptitudes son evaluadas y cuya característica definitoria es la preocupación recurrente por el posible fracaso o mal rendimiento en la tarea y sus consecuencias. Se analizaron las bases conceptuales, características y propiedades psicométricas de: Test Anxiety Scale (Sarason, 1978), Test Anxiety Inventory (Spielberger, 1980), Reactions To Test (Sarason,1984), German Test Anxiety Inventory (Hoddap, 1996), Inventario de Situaciones y Respuestas de Ansiedad (Miguel Tobal \& Cano Vindel, 1988), Cuestionario de Ansiedad en los Exámenes (Valero, 1997) Cognitive Test Anxiety Scale (Cassady \& Jonson, 2002) y Cuestionario de Ansiedad y Rendimiento (Ferrando Varea \& Lorenzo, 2002). Estos instrumentos incluyen entre una y cuatro subescalas, siendo la cognitiva o de preocupación la única presente en todos los inventarios y la que presenta mejores evidencias predictivas respecto al rendimiento académico. Otras escalas como las de emocionalidad, respuestas conductuales y situaciones ansiógenas aportan datos útiles para evaluaciones clínicas. Todos los instrumentos revisados poseen buenos niveles de consistencia interna y validez convergente pero las evidencias sobre su estructura interna son más dispares. Su empleo local requiere en todos los casos de un proceso previo de adaptación a la población estudiantil argentina.
\end{abstract}

Palabras clave: ansiedad ante los exámenes, confiabilidad, validez

\section{Introducción}

La ansiedad ante los exámenes o "test anxiety" puede definirse como una respuesta

\footnotetext{
${ }^{1}$ Por favor dirigir la correspondencia relacionada con este artículo a: Luis Furlan

Laboratorio de Evaluación Psicológica y Educativa.

Facultad de Psicología,

Universidad Nacional de Córdoba

E-mail: luisfurlan@hotmail.com
} 
emocional que las personas presentan en una situación en la cual sus aptitudes son evaluadas. La característica definitoria de tal reacción es la preocupación recurrente por el posible fracaso o mal rendimiento en la tarea y sus consecuencias aversivas para la autoestima, minusvaloración social, y pérdida de algún beneficio esperado (Gutierrez Calvo y Avero 1995)

Se pueden diferenciar el "rasgo" y el "estado" de ansiedad. El primero describe la tendencia a reaccionar emocionalmente con estados ansiosos ante cierto tipo de situaciones, de modo que resulta un patrón estable de respuesta del individuo. El segundo es de carácter transitorio e implica la aparición conjunta de tres sistemas de respuesta que interactúan entre si. Estas son la experiencia subjetiva, la activación fisiológica, y la conducta manifiesta.

Existen numerosas evidencias de la influencia de la ansiedad ante los exámenes en el rendimiento académico, (Culler \& Holahan,1980, Hembree, 1988, Gutierrez Calvo, 1996, Benjamín, Mc Keachie, Lin \& Holinger, 1981, Cassady \& Jonson, 2002) siendo su componente cognitivo (preocupaciones, pensamientos irrelevantes) el que mayor interferencia puede producir sobre las tareas de codificación, organización, almacenamiento y recuperación de información que el estudiante debe realizar cuando se está preparando o rindiendo un examen. Para obtener mediciones estandarizadas de esta variable, concebida como rasgo o como estado se han elaborado diversos cuestionarios aplicables a estudiantes de un amplio rango de edades y niveles de escolaridad. Sus definiciones conceptuales, dimensiones e indicadores han ido variando lo que se ha reflejado en el contenido y formato de los inventarios.

La investigación sistemática en el área se remonta mediados del siglo XX, época en la que Mandler y Sarason (1952) elaboran el Test Anxiety Questionnaire, y existen numerosos inventarios de países anglosajones, algunas adaptaciones al castellano y tres instrumentos de origen Español. No obstante, no hemos encontrado escalas construidas o adaptadas para estudiantes universitarios argentinos. Por este motivo se han utilizado en algunos estudios locales otros cuestionarios más generales como el de Ansiedad Estado-Rasgo STAI (Spielberger, Gorsuch y Lushene, 1972) o el Inventario de Situaciones y Respuestas de Ansiedad ISRA (Miguel Tobal y Cano Vindel, 1986) en el que los exámenes son un ejemplo dentro de las denominadas "situaciones evaluativas". El ISRA cuenta con una adaptación y un baremo realizado en Buenos Aires (Leibovich y Schmidth, 2003) aunque, como se verá más adelante resulta solo parcialmente útil para la evaluación de la ansiedad ante los 
exámenes, dadas sus limitadas propiedades técnicas.

El objetivo de este trabajo es realizar una exhaustiva revisión bibliográfica a fin de: a) identificar los inventarios de ansiedad ante los exámenes (en adelante AE) más relevantes y b) analizar los fundamentos conceptuales y propiedades psicométricas de un conjunto de ellos

\section{Caracterización de los instrumentos}

Se presenta una reseña de los inventarios relevados, por orden histórico de aparición y destacando sus bases conceptuales, características y propiedades psicométricas. Para una descripción más detallada de los índices de confiabilidad y validez se presenta un cuadro comparativo (Cuadro 1) que resume la información más relevante de cada instrumento.

\subsection{Test Anxiety Questionnaire TAQ (Mandler y Sarason, 1952)}

Mandler y Sarason (1952) iniciaron en la Universidad de Yale la investigación empírica sistemática de los problemas de Ansiedad ante los Exámenes acuñando el término test anxiety. Esta fue concebida como una respuesta global y unitaria que incluía manifestaciones cognitivas, somáticas y conductuales. El TAQ que es el primer inventario de autoinforme del que se han encontrado referencias, asume esta unidimensionalidad del constructo y por eso posee una única escala.

Gorsuch (1966) estudió la estructura interna del TAQ mediante análisis factorial y encontró 7 factores de primer orden, dos de segundo y uno de tercer orden, concluyendo que los diferentes tipos de respuestas de ansiedad encontradas en los factores primer orden pueden considerarse como partes de una respuesta total y holística del individuo ante la situación de examen. El valor histórico del TAQ radica en que ha servido como modelo a partir del cual se han desarrollado las nuevas escalas.

A finales de los años 60 surgen también dos de las principales concepciones acerca de la ansiedad que sentarán las bases para la prolífica investigación desarrollada hasta la actualidad. Una de ellas es la distinción entre la ansiedad como estado transitorio y como rasgo estable de la personalidad (Cattell y Scheier, 1961, Spielberger, 1966, 1972) de la que luego surgirá el concepto más refinado de "rasgo específico - situacional" para referirse a una característica personal que se activa selectivamente en ciertas situaciones y promueve la aparición de un estado ansioso intenso. 


\section{Cuadro 1}

Escalas de Ansiedad Ante los Exámenes

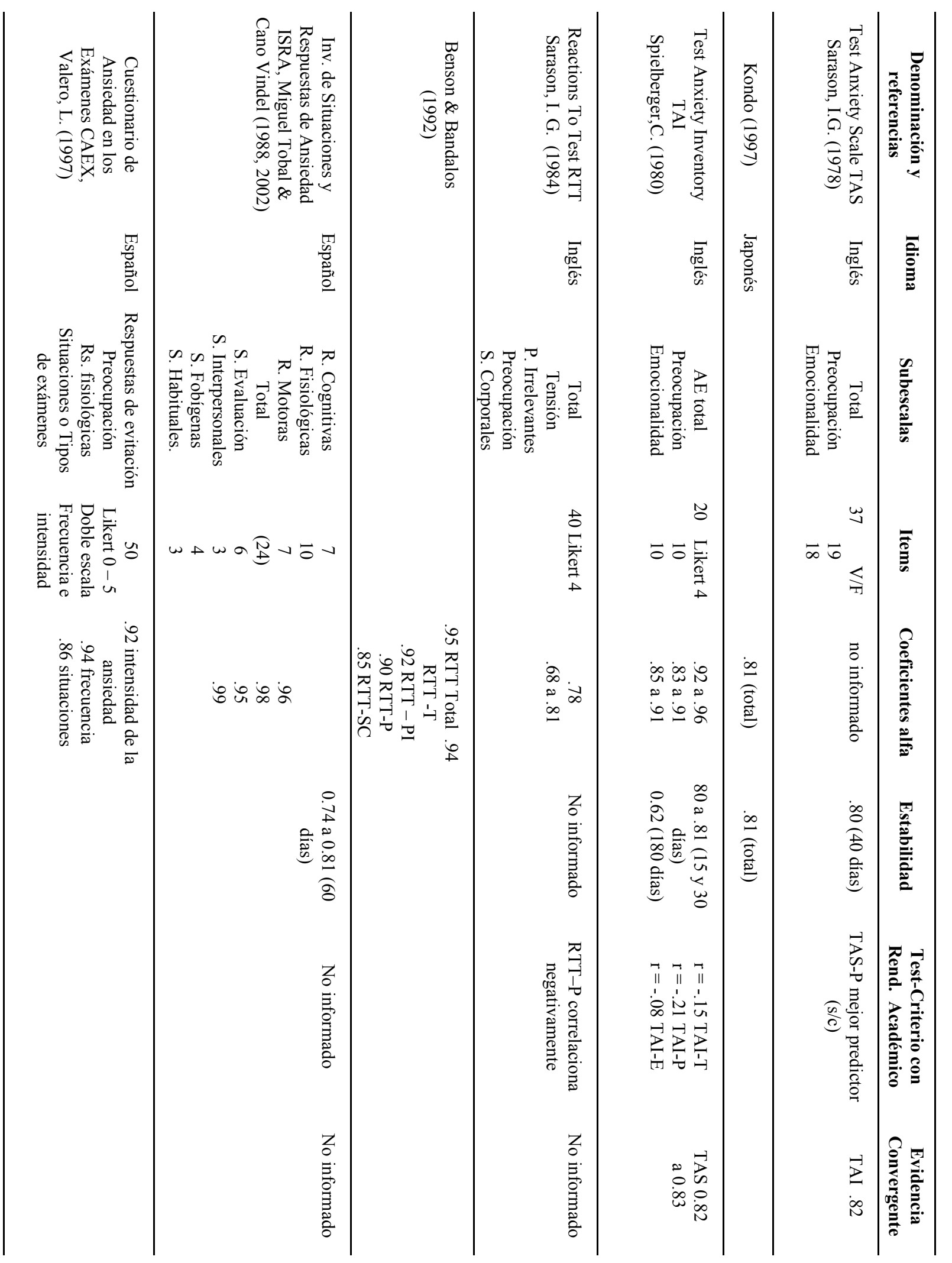




\section{Continuación del Cuadro 1}

Escalas de Ansiedad Ante los Exámenes

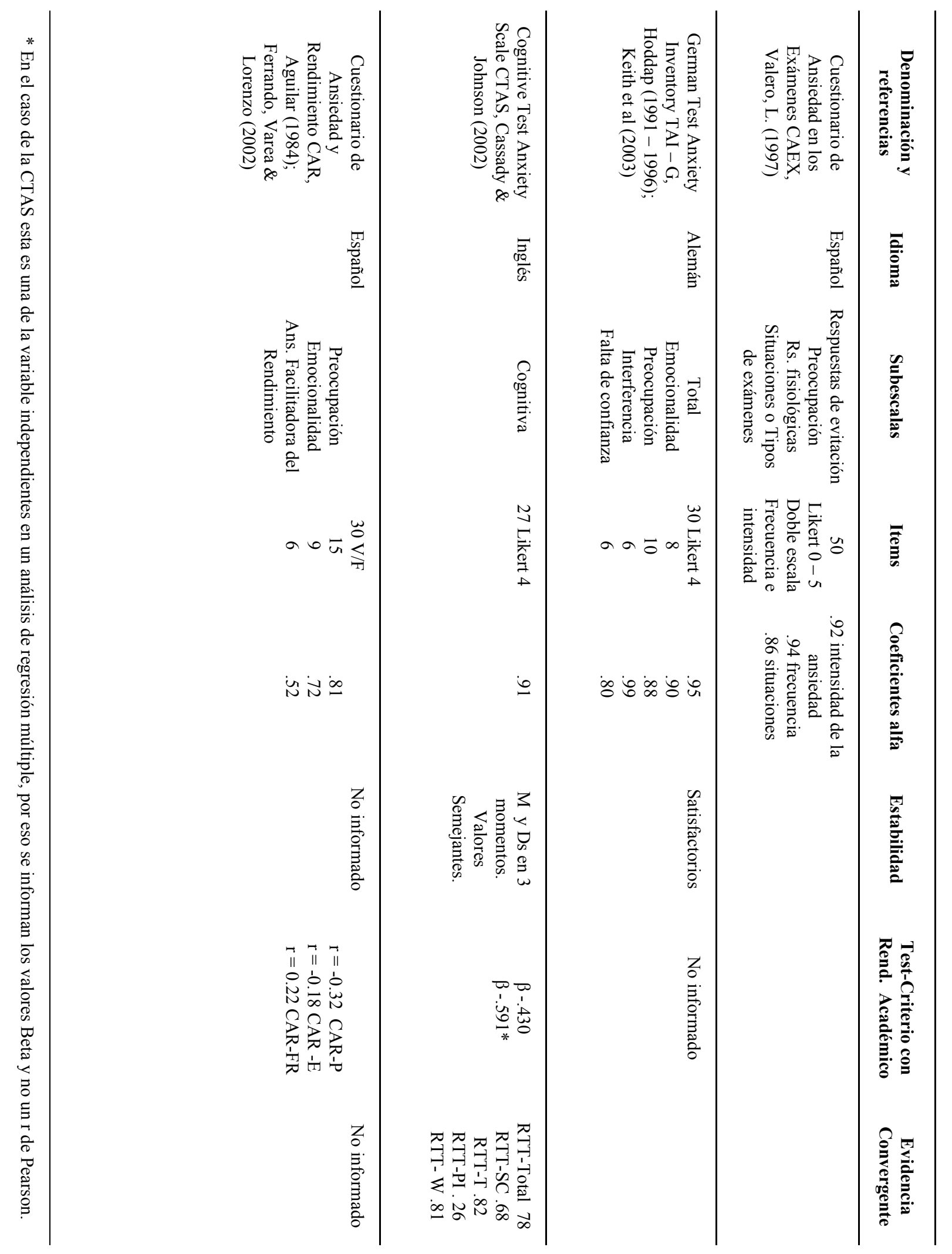


La otra concepción distingue dos dimensiones básicas de la experiencia de ansiedad, una de carácter cognitivo denominada preocupación - "worry”- y otra afectiva llamada emocionalidad - "emotionality"-. El Modelo Dual define la “preocupación” como la focalización de la atención sobre pensamientos irrelevantes para la tarea, tales como las consecuencias de fracasar, la habilidad de los otros en relación ala propia, etc. La "emocionalidad" se refiere a la percepción subjetiva de la activación fisiológica y del estado afectivo que se genera. Este se caracteriza por la tensión, el aumento de la activación, sudoración, frecuencia cardiaca, etc. (Liebert \& Morris, 1972). Los instrumentos psicométricos inspirados en estas concepciones y que mayor trascendencia han tenido fueron la Test Anxiety Scale (Sarason, 1978) y el Test Anxiety Inventory (Spielberger, 1980).

\subsection{Test Anxiety Scale TAS (Sarason, 1978)}

El TAS concibe la AE como un rasgo personal específico y situacional. Esto quiere decir que se considera la existencia de una predisposición relativamente estable y consistente del individuo para reaccionar con determinados niveles de ansiedad ante la situación específica de evaluación. Posee 37 afirmaciones con opciones de respuesta verdadero / falso que conforman las escalas "Preocupación“ y "Emocionalidad", obteniéndose puntuaciones para cada una de ellas y también una general. Incluye manifestaciones de ansiedad en los momentos previos al examen, mientras este se desarrolla y una vez que ha finalizado.

En cuanto a su fiabilidad se han informado niveles satisfactorios en general (Valero, 1999) encontrándose datos más precisos sobre su consistencia interna (alfa .82) en una adaptación al japonés (Kondo, 2002). Para la estabilidad los coeficientes son de .80 en un intervalo de 40 días. Respecto a las evidencias de su validez, las correlaciones test - criterio han sido importantes entre la escala preocupación y el rendimiento académico (Ludlow \& Guida, 1991). El análisis de su estructura interna ha confirmado los dos factores postulados (Richardson et al, 1977).

En diversas oportunidades el TAS ha sido considerado como instrumento bien establecido como para emplearlo en estudios de validez convergente de otras escalas. Además de la adaptación japonesa, se han encontrado referencias a una traducción al español (Serrano Pintado, 2002). El empleo del TAS a partir de los mediados años ochenta aparentemente ha decrecido, lo que puede atribuirse a la aparición de instrumentos más refinados como el Reactios to Test (Sarason, 1984) y/o más breves como la Revised Test 
Anxiety Scale (Benson \& El Zahar, 1994).

\subsection{Test Anxiety Inventory TAI (Spielberger, 1980)}

El TAI es un inventario que al igual que el TAS permite medir la ansiedad de evaluación como un rasgo personal específico - situacional en las dos dimensiones derivadas del Modelo Dual. Posee 20 items con cuatro opciones de respuesta tipo likert que indican la frecuencia con la cuál se experimenta cada reacción. Cada una de las escalas tiene 8 reactivos y otras cuatro que se computan solo en la puntuación total. Para cada dimensión se obtienen puntuaciones parciales.

Su consistencia interna ha sido elevada con alfas superiores a .83 para las subescalas y la puntuación total. Los estudios de estabilidad informan coeficientes de .80 y .81 en dos semanas a un mes y en seis meses de .62. En evidencias de validez convergente con el TAS se han obtenido correlaciones de.82 a .83 (Spielberger, 1980). Para evidencias de validez test-criterio las correlaciones con rendimiento académico fueron de -.21 para el factor preocupación, -.08 para emocionalidad y -.15 para el total (Chapell , Blanding, Silverstein, Takahasi, Newman, Gubi y Mc Cann , 1995).

Ware, Gallasi \& Dew (1990) han señalando aspectos problemáticos no resueltos respecto de la validación del constructo. Algunos ítems resultan problemáticos y los factores W y E presentan cierto grado de solapamiento, con correlaciones entre si de 0,55 a 0,76. Críticas coincidentes se han planteado en general para todos los instrumentos derivados del modelo dual (Ferrando, Varea y Lorenzo, 1999).

Del TAI original se han realizado también adaptaciones al español (Serrano Pintado, 2002) y al hebreo (Zeidner, Nevo \& Lipschitz, 1988) y versiones abreviadas como el Inventario de Autoevaluación de la Ansiedad sobre Exámenes (IDASE) de 8 ítems (Bauermeister, Collazo y Spielberger, 1982) y el TAI - 5 (Taylor \& Deane, 2002) de solo 5 ítems. Spielberger ha sido uno de los principales autores de referencia en este campo durante varios años, dada la trascendencia que el TAI ha tenido a nivel internacional y las reiteradas oportunidades en que se empleó para investigaciones empíricas y estudios de validez convergente, incluso muy recientes (Chapell et al, 1995). A partir de los 80 los instrumentos empiezan a tener otras características, aumentando el número de subescalas para incorporar aportes de nuevas teorías. El cuidado de los aspectos técnicos se incrementa, especialmente con el empleo del Análisis Factorial Confirmatorio para validar los constructos. 


\subsection{Reactions to test RTT (Sarason, 1984)}

El RTT es un inventario que mediante la inclusión de nuevas escalas asimila los aportes conceptuales del modelo de Interferencia Cognitiva (Wine, 1971, Sarason, 1984) Dicho modelo plantea que los estudiantes con elevada ansiedad de examen dirigen su atención hacia pensamientos no relacionados con la evaluación (test-irrelevant thinking), disminuyendo en consecuencia su concentración tanto en la tareas de estudio y preparación como en el momento de enfrentar el examen. En esta línea, la dimensión cognitiva de la $\mathrm{AE}$ se subdivide en dos escalas: "preocupación" y "pensamientos irrelevantes". La emocionalidad se conforma también de dos escalas: la de "tensión" que se refiere a la vivencia subjetiva o sentimiento, y la de "síntomas somáticos" relacionada a indicadores fisiológicos como sudoración, temblor, taquicardia, etc.

En cuanto a su confiabilidad, los índices de consistencia interna han sido satisfactorios para todas las subescalas, que a la vez están moderadamente correlacionadas entre sí, en un rango de .24 a .69 (Sarason, 1984). Posteriormente, Flett, Blankstein y Boas (1987) obtuvieron también intercorrelaciones relativamente bajas o moderadas entre las escalas, lo que sugeriría que se trataría de cuatro dimensiones diferentes.

Sarason analizó también las correlaciones entre las cuatro escalas del RTT y medidas de rendimiento e interferencia cognitiva. La escala de preocupación mostró correlaciones elevadas y negativas con rendimiento y positivas con interferencia. Las otras tres aparecieron no relacionadas. Flett et al (1987) obtuvieron correlaciones elevadas entre el RTT y medidas de temor a la evaluación negativa y ansiedad social, estando solo la escala de pensamientos irrelevantes no relacionada. Alexitch, Blankstein \& Flett (1987) informaron de correlaciones con medidas de expectativas hacia la evaluación (test-taking) pero en este caso los pensamientos irrelevantes tampoco estuvieron relacionados. Esto debilita en parte la evidencia de convergencia del RTT con las otras escalas.

Benson y Bandalos (1992) elaboraron una versión abreviada del RTT con 20 items, que conserva la misma estructura que el original. En el Análisis Factorial Confirmatorio para los factores de primer orden en el caso de la versión original (40 ítems) se obtuvieron indicadores pobres del ajuste del modelo a los datos, aunque para la versión de 20 ítems los índices fueron óptimo (CFI .99, por ejemplo). 
Por otra parte la evidencia empírica apoya la existencia de un factor global de ansiedad ante la evaluación, además de los cuatro de primer orden lo que concuerda con los postulados de Gorsuch (1966).

\subsection{Cuestionario de Ansiedad y Rendimiento CAR (Aguilar, 1984)}

El CAR se basa en el modelo dual (Liebert y Morris, 1972) e incorpora también la noción de ansiedad facilitadora del rendimiento. Alpert y Harber, (1960) señalaron que en los individuos con elevada ansiedad de prueba, en situación de evaluación se activarían dos estados impulsivos de ansiedad. Por una parte se incrementarían la activación autonómica y los pensamientos y preocupaciones, que desviarían la atención, quitándole energía y tiempo para realizar sus tareas. Este fenómeno se denominó "ansiedad perturbadora del rendimiento". Por otra parte se activarían reacciones de "ansiedad facilitadora del rendimiento" estrechamente vinculadas a la resolución de la tarea. Este concepto, también desarrollado por otros autores españoles como Pelechano (1976) y Gutierrez Calvo (1996) acentúa el valor adaptativo de la respuesta de ansiedad. Específicamente, si la percepción de que el nivel del desafío supera el potencial actual para su afrontamiento, se activa un estado de preocupación por la anticipación de un posible fracaso y esto puede movilizar estrategias auxiliares para ampliar los recursos de afrontamiento.

La versión original elaborada por Aguilar (1984) consta de 110 ítems dicotómicos que evalúan las dimensiones Preocupación y Emocionalidad de la Ansiedad Perturbadora del Rendimiento y también la Ansiedad Facilitadora. Es aplicable a niños a partir de los 12 años, con un carácter cognitivo, específico y situacional. Luego de varios estudios se fueron eliminando ítems poco relevantes a los fines de predecir el rendimiento académico positivo o negativo, resultando una versión con 30 ítems referentes a una situación imaginada de evaluación y que intentan reflejar el pensamiento verbal de un sujeto en situación de examen.

Ferrando, Varea \& Lorenzo (2002) realizaron estudios psicométricos de la versión abreviada. Respecto de su consistencia interna se obtuvieron coeficientes alfa de .81 para preocupación (15 items), .72 para emocionalidad (9 items) y .51 para AFR (6 items). La evidencia de estructura interna se estudió mediante el análisis factorial, y se extrajeron tres factores. Preocupación y ansiedad facilitadora son bastante claros, aunque el factor de emocionalidad esta insuficientemente definido. Las correlaciones entre las escalas resultaron altas y positivas entre Preocupación y Emocionalidad y negativas con Ansiedad Facilitadora 
del Rendimiento, algo esperado teóricamente.

En cuanto a la evidencia test-criterio, se tomó como variable a predecir las calificaciones en matemática y como predictoras las puntuaciones totales de cada escala. Se obtuvieron índices moderados para el factor preocupación (-0.32) y emocionalidad (-0.18) siendo el primero el mejor predictor, en concordancia con lo informado por otros autores (Hembree, 1988; Deffenbacher, 1980). Ferrando et al. (2002) sugieren revisar las escalas de emocionalidad y ansiedad facilitadora, de modo de obtener estructuras más puras y poder realizar nuevos estudios de predicción.

\subsection{Inventario de Situaciones y Respuestas de Ansiedad (ISRA) (Tobal \& Cano Vindel,} 1986, 2003)

Este inventario se ha construido en el marco de la Teoría Tridimensional de la Ansiedad (Lang, 1968), de orientación neoconductista, y para evaluar específicamente tres sistemas de respuesta denominados: cognitivo, fisiológico y motor. Esta teoría cuestiona la visión unitaria de la respuesta de ansiedad, dada la escasa correlación encontrada entre los tres sistemas de respuesta. Esta fraccionamiento de la respuesta le restaría valor explicativo al uso genérico del término ansiedad, orientando la evaluación puntualmente hacia los tres sistemas de respuesta y en interacción con situaciones particulares.

Siguiendo el Modelo Multidimensional de Endler (1973), el ISRA evalúa estas respuestas en cuatro grupos de situaciones (de evaluación, interpersonales, con estímulos de carácter fóbico o de la vida cotidiana), permitiendo establecer las áreas más problemáticas para el sujeto y su perfil de reactividad en el triple sistema de respuesta. Este modelo, de carácter interaccionista, cuestiona las explicaciones del comportamiento que atribuyen a los "rasgos" un poder causal, como así también a las que se lo atribuyen al ambiente en forma predominante. Desde esta perspectiva se considera que las manifestaciones puntuales de ansiedad son función, de la interacción que se produce entre un rasgo o predisposición ansiosa y las características de una circunstancia ambiental particular. La influencia de la situación en la conducta del individuo está mediatizada por la percepción que este tiene de la misma, es decir por la interpretación que realiza de la información referente a la situación.

En su versión original del ISRA posee 528 ítems emergentes de la combinación de 24 respuestas agrupadas en los tres Sistemas: Cognitivo (7), Fisiológico (10) y Motor (7), y las 22 situaciones, agrupadas como: evaluación (6), interpersonales (3), fobígenas (4) y 
habituales (3). Miguel Tobal y Cano Vindel (1986) elaboraron posteriormente una versión reducida de 224 ítems, que incluye además una situación abierta descrita por el sujeto y que tiene gran utilidad clínica. Se eliminaron aquellas respuestas consideradas irrelevantes para cada situación particular. Se responde mediante una escala de 0 a 4 (casi nunca a casi siempre) indicando la frecuencia con la que aparece la respuesta ante cada situación. Esto permite obtener puntuaciones parciales para cada subescala de los sistemas de respuesta y también para cada tipo de situación.

Se puede calcular una puntuación total sumando las subescalas, puntaje que indicaría la magnitud del "rasgo", lo que resulta llamativo, teniendo en cuenta los fundamentos de la prueba. Finalmente, las puntuaciones originales se transforman a percentiles, lo que permite una comparación del individuo con la población de referencia.

Respecto de su consistencia interna se obtuvieron alfas superiores a 0,95 para todas las escalas de los sistemas de respuesta. La estabilidad, analizada mediante el método test retest en intervalos de 60 días osciló entre 0,74 y 0,81 para las subescalas y el total. Respecto de su estructura interna, para los sistemas de respuesta se han encontrado los factores inicialmente concebidos, que explican el $52 \%$ de la varianza total. Las situaciones se agrupan en 4 factores que explican el 51,62 \% de la varianza total. El ISRA permite discriminar con bastante precisión a los grupos "normales" de los "clínicos".

Leibovich (2003) ha realizado una adaptación de esta versión para la Argentina modificando algunos aspectos lingüísticos y elaborando baremos para poblaciones clínicas y generales. Richaud \& Sacchi (1995) formulan alguna críticas luego de estudiar la estructura interna del ISRA y comparar los resultados obtenidos en el análisis factorial con la versión original y la abreviada. En la primera obtuvieron los tres factores postulados, siendo el cognitivo el mejor definido. En las situaciones se encontraron las cuatro originales, siendo la de asunción de responsabilidades y evaluación la mejor definida. En la versión breve la estructura resultó menos clara, encontrándose un factor cognitivo - comportamental en el que saturan la mayoría de los ítems de ambos factores. Se encontraron tres situaciones (en lugar de 4), con la interpersonal y de evaluación saturando en un mismo factor.

No obstante estos hallazgos y a pesar de su extensión, el ISRA es ampliamente utilizado en España, particularmente por el grupo de investigadores vinculados a la Sociedad Española de Ansiedad y Estrés. Se lo puede encontrar en varios estudios de caso único, en los que se verifica la eficacia de intervenciones cognitivo-comportamentales. Si se analiza en 
forma estricta, el ISRA contiene en realidad una sola situación, definida como rendir un examen importante, con 2 respuestas cognitivas, 7 fisiológicas y 3 comportamentales. Esto supone una concepción de la Test Anxiety bastante diferente a la que sustenta el resto de los instrumentos, donde el componente cognitivo es mucho más relevante. Con estos ítems se conformaría eventualmente una escala de ansiedad ante los exámenes con 12 afirmaciones. Finalmente, se han encontrado referencias de su empleo en Argentina, en estudios descriptivos desarrollados en la Universidad de San Luis (Correché, Solares; Barbenza y Penna, 2002). Existen datos también sobre una adaptación para Brasil, realizada por AmorimGaudêncio (1998).

\subsection{Cuestionario de Ansiedad ante los Exámenes CAEX (Valero, 1997)}

El CAEX, a diferencia del TAS o el TAI, más que centrarse en el rasgo de ansiedad como constructo, evalúa las características comportamentales de la ansiedad de examen incluyendo respuestas cognitivas, verbales, motoras y fisiológicas y una tipología de los exámenes mas frecuentes. El interés que ha motivado su desarrollo has sido el de reunir en una única prueba todas las manifestaciones de ansiedad que un estudiante puede experimentar frente a los exámenes, a los fines de una evaluación conductual. Por eso también discrimina los niveles de ansiedad subjetiva relativos a la amplia gama de situaciones evaluativas características de las carreras universitarias.

Del análisis de sus 50 ítems emergen cuatro factores, tales como respuestas de evitación, preocupaciones antes o durante el examen, respuestas fisiológicas y situaciones o tipos de examen más frecuentes. Valero (1997) informó coeficientes alfa adecuados para todas las escalas. Según el autor el CAEX es ampliamente utilizado en España fundamentalmente como medida de pre y post en estudios de caso y con grupos sometidos a tratamiento y controles. Su composición permite identificar las áreas más problemáticas en cada caso para planificar tratamientos personalizados.

\subsection{German Test Anxiety Inventory TAI-G (Hoddap 1996)}

A semejanza de la versión original del TAI (Spielberger, 1980) en la adaptación germana se asume la noción de rasgo situacional - específico. No obstante se pueden encontrar varias diferencias entre las dos versiones. El TAI-G se compone de cuatro subescalas (emocionalidad, preocupación, interferencia y falta de confianza) en lugar de dos y posee 30 ítems (en lugar de 20). Esta versión muestra también la influencia del Modelo de 
Interferencia Cognitiva y se vincula al concepto de Autoeficacia desarrollado por Bandura en o relativo a la confianza para afrontar el examen. Otro rasgo distintivo es que sus ítems se refieren a hechos que acontecen solo "durante" la ejecución del examen y se han suprimido las referencias a los momentos previos o posteriores al mismo.

Los aspectos problemáticos con el TAI respecto a la validación del constructo señalados previamente (Ware et al, 1990), motivaron también Hoddap a buscar una versión más rigurosa del Inventario. El TAI - G presenta muy buenos niveles de consistencia interna para todas las subescalas. Respecto de su estructura interna las intercorrelaciones entre escalas fueron moderadas. La estabilidad ha sido analizada en el marco de la Teoría del Rasgo-Estado Latente (LST theory), un enfoque psicométrico desarrollado por Steyer (1987) para determinar si un atributo posee mayormente características de rasgo estable o de estado transitorio. En el Análisis Factorial Confirmatorio realizado por Keith, Hoddap, Shermelleh Engel \& Mossbrugger (2003) se corroboró la existencia de 4 factores de primer orden y uno de segundo orden, con índices de ajuste óptimos. Esta estructura se asemeja a la encontrada en el Reactions to Test, postulando el factor general "ansiedad ante los exámenes".

\subsection{Cognitive Test Anxiety Scale CTAS (Cassady \& Johnson, 2002)}

Las revisiones acerca de la relación entre ansiedad y rendimiento académico (Hembree, 1988, Zeidner, 1998) han demostrado que, entre las diferentes dimensiones de la respuesta de ansiedad, las cogniciones ejercen (más que los aspectos emocionales de activación) una influencia clara e importante sobre el rendimiento. Estas incluyen lo que tradicionalmente se ha denominado "preocupaciones" pero, como se ha visto previamente no se agotan en él (vg. falta de confianza, pensamientos irrelevantes, interferencia). Para evaluar esta dimensión, concebida de un modo más amplio, Cassady \& Johnson (2002) elaboraron la CTAS. Esta escala tiene 27 ítems con cuatro opciones de respuesta que indican la frecuencia de aparición de las reacciones descriptas Se responde con una escala de respuesta que va desde "no es para nada típico o característico en mi", hasta "muy característico o frecuente en mi".

Posee ítems que indican niveles bajos y altos de ansiedad, por lo que las puntuaciones de los primeros deben ser analizadas en forma inversa para el puntaje total y permite clasificar a los sujetos según su nivel de ansiedad (alto, moderado y bajo). Sus ítems se refieren a pensamientos frecuentes en los períodos de preparación de los exámenes donde las 
funciones a desarrollar son las de codificación y almacenamiento de la información y también durante la toma del examen, donde predomina la recuperación la información. Los modelos de Procesamiento de la Información (Naveh-Benjamin, Mc Keachie \& Lin, 1987) han descrito marcadas diferencias entre los estudiantes con elevada y baja ansiedad, respecto de sus habilidades para el estudio y de las estrategias de procesamiento de la información que emplean durante los períodos de preparación para los exámenes.

Si bien la CTAS posee un carácter unidimensional, analizando sus ítems pueden apreciarse diversas facetas de la actividad cognitiva, tales como las valoraciones del propio desempeño y capacidad y sus comparaciones con el de los pares, las preocupaciones por el fracaso y sus consecuencias y las interferencias en la recuperación de información.

Su consistencia interna (alfa .91) es elevada, y en los estudios de test-retest en tres momentos se observó que las medias y desvíos se mantuvieron muy semejantes a pesar de variar los formatos del examen próximo (Cassady, 2001). La evidencia convergente se analizó en sucesivas ocasiones, con el TAI (Spielberger, 1980), el RTT (Sarason, 1984) y la Revised Tests Anxiety Scale ((Benson \& El Zahar, 1994) encontrándose en todos los casos resultados satisfactorios. En algunos estudios de validez test - criterio, con el rendimiento académico como variable dependiente y mediante análisis de regresión múltiple se informó que la CTAS era mejor predictor del rendimiento que los otros predictores incluidos en el modelo, tales como el RTT, el rendimiento previo y la procrastinación (Cassady, 2002).

Si bien se trata de una prueba de reciente elaboración, se han realizado numerosos estudios de sus propiedades psicométricas y se está trabajando en adaptaciones al chino y al árabe (Cassady, Mohammed \& Mathieu, 2004).

\section{Discusión}

Del análisis de los inventarios seleccionados pueden desprenderse algunas primeras conclusiones. La teoría de la Ansiedad Dual, que distingue las dimensiones "preocupación” y "emocionalidad" ha cumplido un papel muy importante en el desarrollo de las investigaciones que relacionan la ansiedad ante los exámenes y el rendimiento académico. El empleo de los instrumentos derivados de esta teoría (el TAS y el TAI) ha sido amplio y se han realizado numerosas adaptaciones para otros contextos culturales y lingüísticos, versiones abreviadas y adaptaciones para poblaciones infantiles. Por otra parte, estos inventarios han sido los modelos a partir de los cuales los nuevos inventarios se han tratado 
de diferenciar, manteniendo algunas de sus características y generando innovaciones.

No obstante ambas escalas han recibido algunas críticas. Ferrando et al (2002) señalan que si bien los análisis estructurales separan los ítems de cada factor con bastante claridad, cuando se analizan diferentes instrumentos con escalas "preocupación" y "emocionalidad" mediante matrices multimétodo- multirasgo no puede establecerse validez discriminante. Aparentemente pesaría más la especificidad del instrumento que la distinción postulada. Además Ferrando et. al (2002) observan también el clásico problema de la escasa validez predictiva de las medidas derivadas de esta teoría y que el rasgo de preocupación sea unidimensional.

Los estudios realizados con versiones modificadas de los instrumentos originales indican que se deberían considerar cuatro factores de primer orden. La estructura del TAI - G ha sido analizada en reiteradas oportunidades confirmándose los factores: emocionalidad, preocupación, interferencia y falta de confianza (Keith, et al., 2003). En el caso del RTT (Benson et al, 1992) también se confirman las escalas: tensión, síntomas corporales, pensamientos irrelevantes y preocupación. En ambos casos se considera también que existe un factor más general, dado que las escalas se correlacionan entre si. La ansiedad ante los exámenes, sería entonces aquella respuesta holística y global postulada en un comienzo por Mandler y Sarason (1952) y ratificada luego por Gorsuch (1966) quien la encontró como factor de segundo orden.

Por otra parte, si también se tiene en cuenta el problema de la fragmentación y falta de sincronía de los tres sistemas de la respuesta ansiosa que plantea el Modelo Tridimensional (Lang, 1968) cabría pensar que un instrumento que refleje la complejidad del fenómeno debe contener escalas para cada una de las dimensiones que si bien pueden diferenciarse y ser relativamente independientes van a compartir también una variabilidad común derivada de la existencia de un factor general de segundo orden. Sin embargo, estimar una puntuación total para el mismo que se derive de la suma o promedio de las puntuaciones de las escalas pareciera discutible. La CTAS se presenta como instrumento de muy buenas propiedades y especialmente útil para estudios de tipo teórico, en los que se de cuenta de las relaciones complejas entre manifestaciones cognitivas de la ansiedad ante los exámenes y rendimiento académico, donde necesariamente participan otras variables y en los que el empleo de modelos de ecuaciones estructurales tiende progresivamente a reemplazar los análisis bivariado. 
Quedaría por revisar tal vez, si en las adaptaciones a otros contextos culturales y lingüísticos esta escala conserva el carácter unidimensional, lo que plantea la necesidad de llevar a cabo análisis factoriales con las nuevas versiones.

Respecto de la faceta comportamental de la respuesta ansiosa pareciera que ha interesado especialmente a los autores españoles (Miguel Tobal y Cano Vindel, 1986., Valero , 1997). Probablemente estos han estado más orientados hacia las aplicaciones clínicas de tipo cognitivo-comportamental, que requieren evaluaciones pormenorizadas y para cada individuo orientadas a la planificación de un tratamiento. Algo semejante ocurre con la inclusión de una variedad de situaciones evaluativas, entendiéndose por ello a los diferentes tipos de exámenes, o incluso el hablar en público, preguntar algo al profesor, etc. Estas solo se encuentran en el ISRA y el CAEX.

El ISRA aparece también como un instrumento preferentemente empleado en investigaciones que ponen a prueba la eficacia de programas de tratamiento en grupos y con estudios de caso. Para estudios específicos en ansiedad ante los exámenes sus desventajas podrían ser su elevado número de ítems y la definición un tanto ambigua de situaciones de evaluación. No obstante, ha sido ampliamente utilizado y como se señaló previamente tiene adaptaciones al inglés, portugués y para países latino americanos. En el caso del CAEX no se han encontrado publicaciones posteriores al artículo en que el autor lo presenta y en las que se haya profundizado en sus propiedades psicométricas.

El CAR es el único inventario de ansiedad ante los exámenes con el que se han realizado estudios psicométricos basados en la teoría de respuesta al ítem (TRI). El trabajo de Ferrando et al (2002) constituye un buen ejemplo de cómo aplicar el modelo TRI en pruebas que miden rasgos latentes. Su punto débil está tal vez en las teorías sustantivas de referencia, poco enriquecidas con los aportes más recientes.

Parece bastante claro que el componente cognitivo de la ansiedad es el que mayor incidencia tiene sobre el desempeño académico tanto en las fases de estudio y preparación como en la situación de examen. Esto obedece a los procesos de interferencia atencional, la concentración en la ejecución, la recuperación de información independientemente de que se originen en un rasgo ansioso preexistente, en el déficit de habilidades o a la interacción de estos factores con otras variables individuales (autoeficacia, por ejemplo) y con características específicas de la situación de examen. Las tendencias recientes conciben la ansiedad ante los exámenes como una variable con múltiples dimensiones: preocupación, 
emocionalidad, interferencia y falta de confianza y a la vez afirman que los alumnos desarrollan estrategias de afrontamiento diferenciales para cada una de ellas en las etapas de preparación y al momento de rendir los exámenes (Stöber y Pekrun, 2004, Keith, Hoddap, Schermelleh-Engel y Moosburguer, 2003).

Otra tendencia es el desarrollo de inventarios para evaluar un rango mayor de emociones asociadas con los exámenes, como por ejemplo el Test Emotions Questionnaire TEQ (Pekrun, Götz, Perry, Hochstadt, Molfenter y Kramer, 2002). Existen estudios interesantes sobre la influencia de la $\mathrm{AE}$ a lo largo del proceso o ciclo de estudio y evaluación (Cassady, 2004) y puede observarse la integración de constructos procedentes de otras perspectivas teóricas (v.g. afrontamiento, evaluación primaria y secundaria, autoeficacia) en la elaboración de los modelos teóricos relacionados (Zohar, 1998).

Finalmente cabe señalar que en nuestro medio se han iniciado recientemente los estudios de adaptación de la Cognitive Test Anxiety Scale (Cassady, 2002), con finalidades de utilización en la población de estudiantes universitarios argentinos.

\section{Referencias}

Benjamín, M., Mc Keachie, W., Lin, Y. \& Holinger, D.(1981). Test Anxiety: Deficits in Information Processing. Journal of Educational Psichology, 73, 6, 816-824.

Benson J. \& Bandalos D. (1992). Second-Order Confirmatory Factor Analysis of the Reactios To Test Scale with Cross Validation. Multivariate behavioral Research, 27, 3, 459-487.

Cassady, J. C. (2004). The impact of cognitive test anxiety on text comprehension and recall in the absence of salient evaluative pressure. Applied Cognitive Psychology, 18, 3, 311-325.

Cassady, J. C. (2004). The influence of cognitive test anxiety across the learning-testing cycle. Learning and Instruction, 14, 6, 569-592.

Cassady, J. C., Mohammed, A., \& Mathieu, L. (2004). Cross-cultural differences in test anxiety: Women in Kuwait and the United States. Journal of Cross-Cultural Psychology, 35, 6, 715-718.

Cassady, J. C. (2001). The stability of undergraduate students' cognitive test anxiety levels. Practical Assessment, Research \& Evaluation, 7, 20. Available online: http://PAREonline.net/getvn.asp. 
Cassady, J. C., \& Johnson, R. E. (2002). Cognitive test anxiety, procrastination, and academic performance. Contemporary Educational Psychology, 27, 270-295.

Chapell M., Blanding, Z., Silverstein, M., Takahasi, M., Newman, B., Gubi, A. y Mc Cann, N. (1995). Test anxiety and academic performance in undergraduate and graduate students. Journal of Educational Psichology, 97, 2, 268-274.

Correché, S., Solares, E., Barbenza, M.y Penna, F., (2002). Situaciones de Ansiedad en Ingresantes a la Universidad Nacional de San Luis. Revista IDEA, 36, 19, 16-23

Culler, R. \& Holahan, C. (1980). Test Anxiety and Academic Performance: The Effects of Study-Related Behaviors Journal of Educational Psichology, 72, 1, 16- 20.

Everson; H.T., Millsap R.E. \& Browne, J.(1989). Cognitive Interference or Skill Deficits: an empitical test of two competing theories of test anxiety. Anxiety Research, 1, 313325.

Ferrando, P, Verea, M \& Lorenzo, U. (1999). Evaluación Psicométrica del Cuestionario de Ansiedad y Rendimiento en una muestra de escolares. Psicothema, 11, 1, 225-236.

Gorsuch, R.L. (1966). The general factor in the Test Anxiety Questionnaire. Psichological Reports, 19, 308

Gutiérrez Calvo, M. y Avero, P. (1995). Ansiedad, estrategias auxiliares y comprensión lectora: déficit de procesamiento vs. falta de confianza. Psicothema, 7, 3, 569-578.

Gutiérrez Calvo, M. (1996). Ansiedad y Deterioro Cognitivo: Incidencia en el rendimiento académico. Revista Ansiedad y Estrés, 2, 3, 173-194.

Hembree, R. (1988). Correlates, causes, and treatment of test anxiety. Review of Educational Research, 58, 47-77.

Hodapp, V. (1996). The TAI-G: A multidimensional approach to the assessment of test anxiety. In C. Schwarzer \& M. Zeidner (Eds.), Stress, Anxiety, and Coping in Academic Settings, 95-130. Tübingen: Francke

Keith, N. Hoddap, V. Shermelleh - Engel, K. y Mossbrugger, H. (2003). Cross Sectional and Longitudinal Confirmatory Factor Models for the Germany Test Anxiety Inventory: A construct Validation. Anxiety, Stress and Coping, 16, 3, 251-270.

Kondo, D. (1997). Strategies for coping with test anxiety. Anxiety, Stress and Coping, 10, 203-215.

Liebert, R.M. y Morris, L.W. (1967). Cognitive and emotional components of test anxiety: a distingtoion and some initial data. Psychological Reports, 20, 975-978. 
Mandler, G and Sarason, S B, (1952). A study of anxiety and learning. Journal of Abnormal and Social Psychology, 47, 166-173.

Mc Ilroy, D. \& Bunting, B. (2002). Personality, Behavior and academic achievement: Principles for educators to inculcate and students to model. Contemporary Educational Psichology, 27, 326-337.

Miguel Tobal, J. \& Cano Vindel, A. (1986). ISRA Inventario de Situaciones y Respuestas de Ansiedad. Tea Ediciones.

Miguel Tobal, J. \& Cano Vindel, A. (2002). ISRA Inventario de Situaciones y Respuestas de Ansiedad. Tea Ediciones.

Naveh-Benjamin, M.; Mc Keachie, W.J. \& Lin, Y. (1987) Two types of test anxious students: support for an information processing model. Journal of Educational Psychology, 79, 131-136.

Pekrun R. (2002). Test anxiety and academic achievement. International Encyclopedia of the Social and Behavorial Sciences, 15610 - 15614.

Pekrun, R., Götz, T., Titz, W. y Perry, R.P. (2002). Academic emotions in student's self regulated learning and achievement: A program of qualitative and quantitative research. Educational Psychologist, 37, 91-106.

Pelechano, V. (1975) Motivación y rendimiento académico. Análisis y modificación de la conducta, 1, 83-110.

Richaud de Minzi, M. \& Sacchi, C. (1995). Estudio de un inventario de situaciones y respuestas de ansiedad con adultos jóvenes argentinos. Revista Interamericana de Psicología, 29, 1, 65-74.

Sarason, I.G. (1978) The Test Anxiety Scale: concept and research. In CD Spielberger \& I.G. Sarason (Eds) Stress and Anxiety, 5, 193-216. Washington DC Hemisphere

Sarason, I G, (1984) Stress, anxiety, and cognitive interference: Reactions to tests. Journal of Personality and Social Psychology, 46, 929-938.

Schwarzer, C. \& Buchwald, P. (2003). Examination Stress: measurement and coping. Anxiety, Stress and Coping, 16, 3, 247-249.

Schwarzer, R. y Jerusalem, M. (1992). Advances in anxiety theorie: a cognitive process approach. In K.A. Hagtvet \& T. B. Jhonsen (Eds) Advances in Test Anxiety Research, 7, 2-31. Lisse, The Netherlands: Swets \& Zeitlinger. 
Spielberger, C.D. (1980). Test Anxiety Inventory Palo Alto, C.A: Consulting Psychologists Press.

Spielberger, C.D. \& Vagg, P. R. (1995). Test Anxiety: a transaccional process model, In Spielberger \& Vagg (Eds). Test Anxiety: Theory, assesment and treatment. Washington: Taylor \& Francis.

Stöber, J. y Pekrun, R. (2004). Advances in test anxiety research. Anxiety, Stress and Doping, $17,3,205-211$.

Valero Aguayo L. (1999). Evaluación de ansiedad ante exámenes: datos de aplicación y fiabilidad de un cuestionario CAEX. Anales de Psicología, 15, 2, 223-231.

Ware, W.B., Gallasy, J.P. y Dew, K.H. (1990). The Test Anxiety Inventory: A confirmatory Factor Analysis. Anxiety Research, 3, 205-212.

Zeidner, M. (1998). Test Anxiety: The State of the Art, Plenum Press, New York.

Zeidner, M., Nevo, B. y Lipschitz, H. (1988). The Hebrew Version of the Test Anxiety Inventory. Haifa: University of Haifa.

Zohar, D. (1998). A additive model of test anxiety: Role of exam-especific expectations. Journal of Educational Psychology, 90, 330-340. 